\title{
The Point Source Method for Inverse Scattering in the Time Domain
}

\author{
D. Russell Luke* $\quad$ Roland Potthast ${ }^{\dagger}$
}

January 30, 2006

\begin{abstract}
Many recent inverse scattering techniques have been designed for single frequency scattered fields in the frequency domain. In practice, however, the data is collected in the time domain. Frequency domain inverse scattering algorithms obviously apply to time-harmonic scattering, or nearly time-harmonic scattering, through application of the Fourier transform. Fourier transform techniques can also be applied to non-timeharmonic scattering from pulses. Our goal here is twofold: first, to establish conditions on the time-dependent waves that provide a correspondence between time domain and frequency domain inverse scattering via Fourier transforms without recourse to the conventional limiting amplitude principle; secondly, we apply the analysis in the first part of this work toward the extension of a particular scattering technique, namely the point source method, to scattering from the requisite pulses. Numerical examples illustrate the method and suggest that reconstructions from admissible pulses deliver superior reconstructions compared to straight averaging of multi-frequency data.
\end{abstract}

Key words. inverse problems, scattering theory, time-domain scattering, image reconstruction

AMS subject classifications. 35R30, 35P25, 94A08

\section{Introduction}

One of the standard problems of inverse scattering is to determine the location and shape of scatterers from measurements of acoustic or electromagnetic waves or fields due to illumination of the region of interest by a given incident wave or field. The mathematical

*Department of Mathematical Sciences, University of Delaware. Email: rluke@math.udel.edu. This author's work was supported in part by a postdoctoral fellowship from the Pacific Institute for the Mathematical Sciences at Simon Fraser University and in part by the Institute for Numerical and Applied Mathematics at the University of Göttingen.

$\dagger$ Institut for Numerical and Applied Mathematics, University of Göttingen, Germany. Web: http://www.scienceatlas.de/nfg. 
theory behind this problem has been thoroughly studied and can be found in such surveys as $[3,11,12,23]$. Frequency domain techniques range from linearized inversion (for example, filtered backprojection), to nonlinear iterative methods (including Newton's method, Landweber iterations, and various manifestations of least-squares fits), to decomposition algorithms (including the dual-space method proposed by Colton-Monk [4,5], the potentialtheoretic technique of Kirsch-Kress [3], and the point-source method of Potthast [21]) to linear sampling and probe methods (including the linear sampling method of Colton and Kirsch [2], the factorization method of Kirsch [8], the probe and enclosure methods of Ikehata [7], the method of singular sources [21], the no response test of Luke and Potthast [16], and the range test of Potthast, Sylvester and Kusiak [22]). While the methodologies are diverse, they all focus on single frequency, time-harmonic waves. Insofar as these techniques can be applied to one frequency, they can be applied to all - with the possible exception of the zero frequency. Linearized inversion techniques like filtered backprojection which employ the Born or physical optics approximations are limited to high frequency scattering. In contrast, none of the other techniques mentioned above have such limitations. Moreover, they work particularly well in the resonance region, that is, at low frequencies, where the scatterers are about the same size as the wavelength of the incident field. The extension of these methods to multifrequency settings is often implicit.

In this work we consider time-dependent nonharmonic waves, for which the multifrequency content of the wave must be explicitly taken into account. In particular, we study plane wave pulses with a frequency content satisfying certain decay/support conditions for large and small frequencies. The conventional limiting amplitude principle does not apply in this setting. Indeed the limiting amplitude principle yields the steady state solution to the inhomogeneous wave equation with zero initial and boundary values in terms of radiating solutions to the inhomogeneous Helmholtz equation with zero boundary values, that is, in terms of time-harmonic fields. For the case we consider here, the steady state is not very interesting - it is just zero. We must therefore reconstruct the fields for all time, or, in practical terms, from the time the pulse is emitted to the time the wave has passed outside of some measurement sphere in the far field of the scatterer.

Since the inverse scattering methods mentioned above are essentially frequency-domain techniques, the first part of this paper, section 2 , is concerned with precisely prescribing the types of nonharmonic waves that are amenable to frequency domain treatment. Ultimately, we will apply the Fourier transform to the measured time-dependent fields, and solve the inverse scattering problem pointwise in the frequency domain using any of the techniques listed above. The solution to the full inverse scattering problem is a weighted average of the single-frequency solutions, depending on the amplitudes of the frequencies in the timedependent pulse. We take some care to establish an appropriate space of the functions in which to work in the time-domain. This amounts to the determination of decay conditions on the scattered waves in time and space that are the nonharmonic, time-domain counterpart to the Sommerfeld radiation condition. We explicitly formulate these conditions and prove equivalence of the family of frequency-domain boundary value problems with scattering in the time-domain from the requisite pulses. 
In the second part of the paper, section 3 , we apply these results to a particular inverse scattering technique, namely the point-source method. We determine in what sense the point-source method converges in the time domain, and establish results on the regularity of the method, which is essential for ill-posed inverse problems. The original point-source method is a scheme to reconstruct time-harmonic waves, which satisfy the Sommerfeld radiation condition, from their far field patterns. At the heart of the method is the construction of a density $g$ which is used to backproject the data from the far field to some subset $E$ of the near field of the scatterer:

$$
u^{s}(x ; \kappa)=\int_{\mathbb{S}} e^{-i \kappa x \cdot \hat{y}} g(\hat{y} ; \kappa) u^{\infty}(-\hat{y} ; \kappa) d s(\hat{y}), \quad x \in \mathbb{E},
$$

where $u^{\infty}$ is the far field pattern and $u^{s}$ is the reconstructed scattered field. For nonharmonic waves, the dependency of $g$ on $\kappa$ is crucial to the convergence of the reconstruction. We restrict ourselves, for the most part to the case where the incoming wave has no DC content, that is, the wavenumbers in the pulse are supported on $\mathbb{R} \backslash\{0\}$. Lemma 3.6 and Theorem 3.7, which establish the regularity of the point source method in the time domain, make the further assumption that the pulses are bandlimited, that is, the frequencies are supported on compact sets of $\mathbb{R} \backslash\{0\}$. We then obtain uniform bounds for $g$ with respect to $\kappa$, which carries over to the reconstructed fields. The exclusion of the zero frequency is a technical condition that arises only in two dimensional settings. In two dimensions the fundamental solution has a strong singularity with respect to the wave number at $\kappa=0$ which we avoid by exclusion of a neighborhood of $\kappa=0$. For practical applications in acoustics, it is not necessary to burden oneself with such technicalities since zero frequency acoustic waves are not physical. We provide appropriate conditions for the fields under consideration to obtain either convergence in norm or point-wise convergence in time.

We conclude this work in section 4 with numerical results for the method in the time domain. We show that the time-domain method with admissible pulses results in a weighted average of multifrequency data that is not implicit in arbitrary multi-frequency data. We compare reconstructions from the time-domain weighted averages with reconstructions from unweighted multifrequency data to illustrate the superiority of reconstructions from admissible pulses.

\section{Forward Scattering}

We begin with waves traveling through a homogeneous, isotropic medium with an inclusion denoted by $\Omega \subset \mathbb{R}^{m}, m=2,3$. These are modeled with the homogeneous wave equation

$$
\left(\triangle-\frac{n^{2}}{c^{2}} \frac{\partial^{2}}{\partial t^{2}}\right) V(x, t)=0
$$

where $x \in \mathbb{R}^{m}$ is the spatial variable, $t \in \mathbb{R}$ denotes time, $V: \mathbb{R}^{m} \times \mathbb{R} \rightarrow \mathbb{R}, n=$ const $>0$ and $\triangle$ denotes the spatial Laplacian

$$
\triangle V(x, t)=\left(\frac{\partial^{2}}{\partial x_{1}^{2}}+\cdots+\frac{\partial^{2}}{\partial x_{m}^{2}}\right) V(x, t), \quad x=\left(x_{1}, \ldots, x_{m}\right) \in \mathbb{R}^{m}, t \in \mathbb{R} .
$$


If the waves are time-harmonic, then the wave $V$ takes the form $V(x, t, \omega)=$ $\operatorname{Re}\{v(x, \omega) \exp [i \omega t]\}$ where $\omega$ is the frequency of the wave. The spatial component of the wave, $v(\cdot, \omega)$, satisfies the Helmholtz equation

$$
\left(\triangle+k^{2} n^{2}\right) v(x, \omega)=0 .
$$

where $k=\omega / c=2 \pi / \lambda$ is the wave number, and $\lambda$ is the wavelength. Without loss of generality, we consider only a normalized $(c=1)$, nondimensionalized wave equation in freespace $\left(n=1\right.$ on $\bar{\Omega}^{c}$, the complement of $\Omega$ ) and write the frequency variable as $\kappa$ rather than $\omega$.

Very little changes when the time-harmonic assumption is dropped, however we need some further assumptions on the behavior of the waves. If we assume that, for $x$ fixed, $V(x, \cdot) \in$ $L^{2}(\mathbb{R})$ and that the wave and its first derivative with respect to time decay sufficiently fast as time approaches infinity, $V(x, t)$ and $\frac{\partial}{\partial t} V(x, t) \rightarrow 0$ as $|t| \rightarrow \infty$, then taking the Fourier transform with respect to time of both sides of Eq.(2.1) yields Eq.(2.2). Here $v(x, \kappa)=$ $\left(\mathcal{F}_{t} V\right)(x, \kappa)$ where

$$
\left(\mathcal{F}_{t} V\right)(x, \kappa):=\frac{1}{\sqrt{2 \pi}} \int_{-\infty}^{\infty} e^{-i \kappa t} V(x, t) d t
$$

and

$$
\left(\mathcal{F}_{\kappa} v\right)(x, t):=\frac{1}{\sqrt{2 \pi}} \int_{-\infty}^{\infty} e^{i \kappa t} v(x, \kappa) d k .
$$

Note that $\mathcal{F}_{\kappa} \mathcal{F}_{t} V=V$.

\subsection{The frequency domain}

The time-domain wave is a real-valued mapping $V: \mathbb{R}^{m} \backslash \Omega \times \mathbb{R} \rightarrow \mathbb{R}$. Thus, its Fourier transform is a Hermitian function of the frequency $\kappa \in \mathbb{R}$, that is it satisfies the property $v(x, k)=\overline{v(x,-k)}$ where $\bar{v}$ denotes the complex conjugate of $v$. In this case, the results surveyed below hold for all wave numbers $\kappa \neq 0$. Throughout this work, the obstacle is described by the bounded domain $\Omega \subset \mathbb{R}^{m}$ ( $m=2$, or 3 ) with a connected, $C^{2}$ (twice continuously differentiable) boundary $\partial \Omega$ with outward unit normal $\nu$. More general, piecewise $C^{2}$ boundaries are also possible (see [6]).

For a fixed $\kappa$, given a continuous function $f(\cdot, \kappa): \partial \Omega \rightarrow \mathbb{C}$, we seek the field $v(\cdot, \kappa) \in$ $C^{2}\left(\bar{\Omega}^{c}\right) \cap C\left(\Omega^{c}\right)$ that satisfies the Helmholtz equation with one of the following boundary conditions:

$$
\begin{aligned}
&\left(\triangle+\kappa^{2}\right) v(x, \kappa)=0, \quad x \in \bar{\Omega}^{c}, \\
& v(x, \kappa)=f(x, \kappa), \quad x \in \partial \Omega \quad \text { (sound-soft obstacle), } \\
& \frac{\partial v(x, \kappa)}{\partial \nu(x)}=f(x, \kappa), \quad x \in \partial \Omega \quad \text { (sound-hard obstacle), } \\
& \frac{\partial v(x, \kappa)}{\partial \nu(x)}+i \lambda(\kappa) v(x, \kappa)=f(x, \kappa), \quad x \in \partial \Omega
\end{aligned}
$$

(impedance obstacle with $\lambda: \mathbb{R} \rightarrow \mathbb{R}_{+}$). 
For a fixed $\kappa$, a solution $v(\cdot, \kappa)$ to Eq. $(2.3)$ whose domain of definition contains the exterior of some sphere is called radiating if it satisfies the Sommerfeld Radiation Condition:

$$
|x|^{\frac{m-1}{2}}\left(\frac{\partial}{\partial|x|}-i \kappa\right) v(x, \kappa) \rightarrow 0, \quad|x| \rightarrow \infty,
$$

uniformly in all directions.

The frequency-domain scattering problem that is central to this work is stated as follows.

Let the sound-soft scatterer $\Omega$ be embedded in a homogeneous medium. Given a single-frequency incident field $v^{i}(\cdot, \kappa): \mathbb{R}^{m} \rightarrow \mathbb{C}$ that solves Eq.(2.3) on all of $\mathbb{R}^{m}$, find the total field $v(\cdot, \kappa): \Omega^{c} \rightarrow \mathbb{C}$ satisfying Eq.(2.3) on $\bar{\Omega}^{c}$ with $f:=0$ in Eq.(2.4), and with $v=v^{i}+v^{s}$, where $v^{s}(\cdot, \kappa)$ is the scattered field satisfying Eq.(2.3) and the radiation condition Eq.(2.7).

For simplicity, we only treat problems with the Dirichlet boundary condition Eq.(2.4). The other boundary conditions Eq.(2.5)-(2.6) are handled similarly.

It is well known, that the scattering problem has a unique solution [3]. The scattered field $v^{s}$ is a radiating solution to Eq.(2.3) and has the asymptotic behavior

$$
v^{s}(x, \kappa)=\frac{e^{i \kappa|x|}}{|x|^{\frac{(m-1)}{2}}}\left\{v^{\infty}(\hat{x}, \kappa)+O\left(\frac{1}{|x|}\right)\right\},|x| \rightarrow \infty,
$$

where the function $v^{\infty}(\cdot, \kappa): \mathbb{S} \rightarrow \mathbb{C}$ is known as far field pattern,

$$
\mathbb{S}:=\left\{x \in \mathbb{R}^{m}|| x \mid=1\right\} \quad \text { and } \quad \hat{x}:=\frac{x}{|x|} .
$$

Green's formula, stated below, represents fields in terms of fundamental solutions to the Helmholtz equation. In particular, let $\Omega \subset \mathbb{R}^{m}$ and $v(\cdot, \kappa) \in C^{2}\left(\bar{\Omega}^{c}\right) \cap C\left(\Omega^{c}\right)$ satisfy Eq.(2.3) and Eq.(2.7) (i.e., $v$ is a radiating solution to the Helmholtz equation), with normal derivative on $\partial \Omega$ in the sense of Gâteaux. Then

$$
v(x, \kappa)=\int_{\partial \Omega}\left\{v(y, \kappa) \frac{\partial \Phi(y, x, \kappa)}{\partial \nu(y)}-\frac{\partial v}{\partial \nu}(y, \kappa) \Phi(y, x, \kappa)\right\} d s(y), \quad x \in \bar{\Omega}^{c},
$$

where $\Phi(\cdot, x, \kappa)$ is the free space fundamental solution to Eq.(2.3) in $\mathbb{R}^{m} \backslash\{x\}$. To accommodate negative frequencies, the fundamental solution is defined so that it is a Hermitian function of $\kappa$ :

$$
\begin{aligned}
& \kappa>0 \quad \Phi(x, z, \kappa) \equiv \begin{cases}\frac{i}{4} H_{0}^{(1)}(\kappa|x-z|), & x \neq z, \text { and } m=2 \\
\frac{1}{4 \pi} \frac{e^{i k|x-z|}}{|x-z|}, & x \neq z, \text { and } m=3,\end{cases} \\
& \kappa<0 \quad \Phi(x, z, \kappa) \equiv \begin{cases}\frac{-i}{4} H_{0}^{(2)}(|\kappa||x-z|), & x \neq z, \text { and } m=2 \\
\frac{1}{4 \pi} \frac{e^{-i|k||x-z|}}{|x-z|}, & x \neq z, \text { and } m=3,\end{cases}
\end{aligned}
$$

where $H_{0}^{(n)}$ denotes the zero-th order Hankel function of the $n$-th kind [3, Eq.(3.60) and $\mathrm{Eq}(2.1)]$. 
Remark 2.1 Note that in three-dimensions there is no need to exclude $\kappa=0$, however in two dimensions the Hankel function has a singularity at the origin which we avoid by excluding a neighborhood of $\kappa=0$. In acoustic scattering zero-frequency waves are not physical so the exclusion of the origin does not warrant undue attention.

The representation in Eq.(2.9) gives the radiating solution to the Helmholtz equation as a combination of acoustic single- and double-layer potentials defined respectively by

$$
\left(\mathcal{S}_{\kappa} \varphi\right)(x, \kappa)=\int_{\partial \Omega} \Phi(x, y, \kappa) \varphi(y) d s(y) \quad x \in \mathbb{R}^{m} \backslash \partial \Omega
$$

and

$$
\left(\mathcal{K}_{\kappa} \varphi\right)(x, \kappa)=\int_{\partial \Omega} \frac{\partial \Phi(x, y, \kappa)}{\partial \nu(y)} \varphi(y) d s(y) \quad x \in \mathbb{R}^{m} \backslash \partial \Omega .
$$

Assuming that $v$ is a solution to the scattering problem for a sound-soft scatterer with incident wave $v^{i}$ satisfying Eq.(2.3) on all of $\mathbb{R}^{m}$, then the first part of the integrand in Eq.(2.9) is zero and we have $v(x, \kappa)=v^{i}(x, \kappa)+v^{s}(x, \kappa), \quad x \in \bar{\Omega}^{c}, \quad \kappa \in \mathbb{R} \backslash\{0\}$, where the scattered field is given by Huygens' principle as

$$
v^{s}(x, \kappa)=-\int_{\partial \Omega} \frac{\partial v(y, \kappa)}{\partial \nu(y)} \Phi(x, y, \kappa) d s(y), \quad x \in \bar{\Omega}^{c} .
$$

The corresponding far field pattern is given by

$$
v^{\infty}(\hat{x}, \kappa)=-\gamma \int_{\partial \Omega} \frac{\partial v}{\partial \nu}(y, \kappa) e^{-i \kappa \hat{x} \cdot y} d s(y), \quad \hat{x} \in \mathbb{S},
$$

which follows by passing to the far field pattern of $\Phi(\cdot, y, \kappa)$.

The exterior Dirichlet problem is the fundamental problem we address in what follows. This is stated precisely as:

Given a continuous function $f$ on $\partial \Omega$, find a radiating solution $v \in C^{2}\left(\bar{\Omega}^{c}\right) \cap$ $C\left(\Omega^{c}\right)$ to Eq.(2.3) that satisfies Eq.(2.4).

The following results, which we state without proof, are well known.

Theorem 2.2 For $\kappa \in \mathbb{R} \backslash\{0\}$ fixed, the exterior Dirichlet problem has a unique solution that depends continuously on the boundary data with respect to uniform convergence of the solution on $\Omega^{c}$ and all its derivatives on closed subsets of $\bar{\Omega}^{c}$.

Corollary 2.3 If the boundary value $f(x, \kappa)$ is $n$-times smoothly differentiable with respect to $\kappa \in \mathbb{R} \backslash\{0\}$, then so is the corresponding solution to the exterior Dirichlet problem. Likewise, if $f(x, \kappa)$ is Hermitian with respect to $\kappa \in \mathbb{R} \backslash\{0\}$, then so is the corresponding solution to the exterior Dirichlet problem. 
Corollary 2.4 Let $f \in C(\partial \Omega) \times C(\mathbb{R} \backslash\{0\})$ be the boundary value for the exterior Dirichlet problem parameterized by $\kappa \in \mathbb{R} \backslash\{0\}$. Then the corresponding solution $v(x, \kappa)$ exists and satisfies the radiation condition Eq.(2.7) uniformly in $\kappa$ on compact subsets of $\mathbb{R} \backslash\{0\}$.

Proof. Since $f$ is continuous, Theorem 2.2 guarantees that $v(x, \kappa)$ exists pointwise in $\kappa$, and, by definition, satisfies Eq. (2.7) pointwise in $\kappa$. Moreover, by Corollary $2.3 v$ is continuous in $\kappa$ on $\mathbb{R} \backslash\{0\}$, therefore $v$ satisfies Eq.(2.7) uniformly in $\kappa$ on compact subsets of $\mathbb{R} \backslash\{0\}$.

\subsection{The time-domain}

We now turn our attention to scattering in the time-domain, where the waves $V: \mathbb{R}^{m} \times \mathbb{R} \rightarrow$ $\mathbb{R}$ satisfy the wave equation Eq.(2.1) with $n=c=1$ on $x \in \bar{\Omega}^{c}$, that is,

$$
\begin{aligned}
\left(\triangle-\frac{\partial^{2}}{\partial t^{2}}\right) V(x, t) & =0, \quad x \in \bar{\Omega}^{c}, \\
V(x, t) & =F(x, t), \quad \forall t \text { and } x \in \partial \Omega \quad \text { (sound-soft) }
\end{aligned}
$$

Radiating solutions to the wave equation whose domain of definition contains the exterior of some sphere satisfy the time-domain Sommerfeld Radiation Condition:

$$
\left\||x|^{\frac{m-1}{2}}\left(\frac{\partial}{\partial|x|}-\frac{\partial}{\partial t}\right) V(x, \cdot)\right\|_{L^{2}(\mathbb{R})} \rightarrow 0, \quad|x| \rightarrow \infty
$$

uniformly in all directions.

Remark 2.5 Note that, in contrast to the frequency domain radiation condition Eq.(2.7), the radiation condition Eq.(2.18) is satisfied in norm rather than pointwise with respect to $t$. Also notice that no initial value is specified in the system above.

The boundary-value problem Eq.(2.16)-(2.18) is under-determined. To remedy this we impose further restrictions on the decay of $V$ and its derivatives. Using multi-index notation, these are given explicitly by

$$
\frac{\partial^{n}}{\partial t^{n}} \frac{\partial^{|l|}}{\partial x^{l}} V(x, \cdot) \in L^{1}(\mathbb{R}), \quad|l|, n \in\{0,1,2\},
$$

and

$$
\int_{|t|>r}\left|\frac{\partial^{n}}{\partial t^{n}} \frac{\partial^{|l|}}{\partial x^{l}} V(x, t)\right| \rightarrow 0, \quad r \rightarrow \infty, \quad|l|, n \in\{0,1,2\},
$$

uniformly in $x$ on compact sets, where $l$ is a multi-index. The motivation for these decay conditions will become apparent in the proof to the correspondence between the time-domain scattering problem and the frequency domain problem, Theorem 2.7. 
Remark 2.6 We would like to point out the contrast between the system of equations Eq.(2.16)-(2.18) together with the decay conditions Eq.(2.19)-(2.20) and systems for which the limiting amplitude principle [17] holds. The limiting amplitude principle gives the steady state solution to the inhomogeneous wave equation with zero initial and boundary values in terms of radiating solutions to the inhomogeneous Helmholtz equation with zero boundary values. This is very different from the situation above, where, rather than the steady state, we are interested in the behavior of the waves for all time. Indeed, the steady state for our system of equations is not very interesting since it is simply zero. Instead, it is what happens in between that allows us to reconstruct the obstacle.

The time-domain Dirichlet scattering problem that we consider is the following.

For a scatterer $\Omega$, given an incident field $V^{i}: \mathbb{R}^{m} \times \mathbb{R} \rightarrow \mathbb{R}$ that solves Eq.(2.16) on all of $\mathbb{R}^{m} \times \mathbb{R}$, find the total field $V: \Omega^{c} \rightarrow \mathbb{R}$ satisfying Eq.(2.16) on $\bar{\Omega}^{c}$ with $F:=0$ in Eq.(2.17) and with $V=V^{i}+V^{s}$, where $V^{s}$ is the scattered field satisfying Eq.(2.16) and the radiation conditions Eq.(2.18)-(2.20).

The next theorem establishes the correspondence between this scattering problem and the classical scattering problem in the frequency-domain.

Lemma 2.7 (Correspondence of time- and frequency-domain.) Let $f(x, \kappa): \partial \Omega \times \mathbb{R} \rightarrow \mathbb{C}$ satisfy $f \in C(\partial \Omega) \times C^{2}(\mathbb{K})$, with $f(x, \cdot)$ Hermitian and $\operatorname{supp} f=$ $\partial \Omega \times \mathbb{K}$, where $\mathbb{K} \subset \mathbb{R} \backslash\{0\}$ is compact. Then the function $v$ satisfies the exterior Dirichlet problem at almost every $\kappa \in \mathbb{K}$ if and only if the Fourier dual $V: \mathbb{R}^{m} \times \mathbb{R} \rightarrow \mathbb{R}, V \in$ $C^{2}\left(\bar{\Omega}^{c}\right) \times C^{2}(\mathbb{R})$, satisfies the time-domain boundary value problem Eq.(2.16)-(2.20), with $F(x, t):=\left(\mathcal{F}_{\kappa} f\right)(x, t)$ in Eq.(2.17).

Proof. Suppose that $v(x, \kappa)$ satisfies the exterior Dirichlet problem with boundary values $f$ at almost every $\kappa \in \mathbb{K}$. Then, by Corollary 2.3, $v$ is almost everywhere equivalent to $v_{*} \in\left(C^{2}\left(\bar{\Omega}^{c}\right) \cap C\left(\Omega^{c}\right)\right) \times C^{2}(\mathbb{R})$, with $\operatorname{supp} v_{*}(x, \cdot) \subset \mathbb{K}$ and $v_{*}(x,-k)=\overline{v_{*}(x, k)}$. Also, by Corollary $2.4, v_{*}$ satisfies Eq.(2.7) uniformly in $\kappa$ on $\mathbb{K}$, thus

$$
\left\||x|^{\frac{m-1}{2}}\left(\frac{\partial}{\partial|x|}-i \kappa\right) v_{*}(x, \cdot)\right\|_{L^{2}(\mathbb{R})} \rightarrow 0, \quad|x| \rightarrow \infty .
$$

We show next that the Fourier transform of $v_{*}$ and it's derivatives are absolutely integrable with respect to $\kappa$ uniformly in $\mathrm{x}$ on compact sets. To see this, recall that, for $|l|=0,1$ or 2 , the partial derivative $\frac{\partial^{|l|} v_{*}}{\partial x^{l}}$ is Hermitian and belongs to $\left(C^{2-|l|}\left(\bar{\Omega}^{c}\right) \cap C\left(\Omega^{c}\right)\right) \times C^{2}(\mathbb{R})$. The Fourier transform of $\frac{\partial^{|l|} v_{*}}{\partial x^{l}}$, call it $V_{l}$ where $V_{l}(x, t)=\left(\mathcal{F}_{\kappa} \frac{\partial^{|l|} v_{*}}{\partial x^{l}}\right)(x, t)$, is therefore a bounded, real-valued function with $\mathcal{F}_{\kappa}(i \kappa)^{n} \frac{\partial^{|l|} v_{*}}{\partial x^{l}}=\frac{\partial^{n} V_{l}}{\partial t^{n}}$, satisfying $\left|\frac{\partial^{n} V_{l}(x, t)}{\partial t^{n}}\right| \leq M_{|l|}(x)(1+|t|)^{-2}$ by standard Fourier analysis, and, in particular, $\frac{\partial^{n} V_{l}(x, \cdot)}{\partial t^{n}} \in L^{1}(\mathbb{R})$, for $n \geq 0$ uniformly in $\mathrm{x}$ on compact sets. Now, since $v_{*}$ is bandlimited with respect to $\kappa$ and twice continuously differentiable with respect to $x$ then $\frac{\partial^{|l|} v_{*}}{\partial x^{l}} \in L^{1}(\mathbb{R})$ uniformly in $x$ on compact sets. Thus, by $[9$, Theorem 53.5] we can differentiate under the integral in the Fourier transform to 
yield $V_{l}=\frac{\partial^{|l|} V}{\partial x^{l}}(|| l \mid=0,1$ or 2$)$. The Fourier dual $V$ therefore satisfies Eq.(2.19)-(2.20) and the boundary value problem Eq.(2.16)-(2.18) where the radiation condition Eq.(2.18) follows from Parseval's relation.

Conversely, suppose that the bounded, real-valued function $V: \mathbb{R}^{m} \times \mathbb{R} \rightarrow \mathbb{R}, V \in$ $C^{2}\left(\bar{\Omega}^{c}\right) \times C^{2}(\mathbb{R})$, satisfies Eq.(2.19)-(2.20) uniformly in $x$ on compact sets, in addition to the time-domain boundary value problem Eq.(2.16)-(2.18), with $F(x, t):=\left(\mathcal{F}_{\kappa} f\right)(x, t)$ in Eq.(2.17). Then [9, Theorem 53.5] can be applied to show $\mathcal{F}_{t} \frac{\partial V}{\partial|x|}=\frac{\partial v}{\partial|x|}$ and $\mathcal{F}_{t} \triangle V=\triangle v$, where $v=\mathcal{F}_{t} V$. These, together with the decay conditions Eq.(2.20) yield

$$
\begin{aligned}
\left(\triangle+\kappa^{2}\right) v(x, \kappa) & =0, \quad x \in \bar{\Omega}^{c}, \\
v(x, \kappa) & =f(x, \kappa), \quad x \in \partial \Omega, \\
\text { and }\left\||x|^{\frac{m-1}{2}}\left(\frac{\partial}{\partial|x|}-i \kappa\right) v(x, \cdot)\right\|_{L^{2}(\mathbb{R})} & \rightarrow 0, \quad|x| \rightarrow \infty .
\end{aligned}
$$

To complete the proof, we must achieve a pointwise decay condition from Eq.(2.22). To do this, define $\mathbb{S}_{j}:=\left\{x \in \mathbb{R}^{m}|| x \mid=r_{j}\right\}$, where the sequence of scalars $r_{j} \rightarrow \infty$. The corresponding sequence of functions is given by

$$
\psi_{j}(\kappa):=\left.\max _{x \in \mathbb{S}_{j}}|| x\right|^{\frac{m-1}{2}}\left(\frac{\partial}{\partial|x|}-i \kappa\right) v(x, \kappa) \mid .
$$

The radiation condition Eq.(2.22) implies that $\left\|\psi_{j}\right\|_{L^{2}} \rightarrow 0$, thus there exists a subsequence $\psi_{j_{i}}(\kappa)$ converging pointwise almost everywhere to zero in $\kappa[24$, Theorem 3.12]. Since $v(\cdot, \kappa)$ satisfies Eq. $(2.3), \psi_{j_{i}}(\kappa) \rightarrow 0$ implies that

$$
\left.|| x\right|^{\frac{m-1}{2}}\left(\frac{\partial}{\partial|x|}-i \kappa\right) v(x, \kappa)|\rightarrow 0, \quad| x \mid \rightarrow \infty,
$$

that is, $v(x, \kappa)$ satisfies the exterior Dirichlet Problem at almost every $\kappa$.

Theorem 2.8 (Uniqueness and Existence for time-domain scattering.) Under the hypotheses of Lemma 2.7, solutions $V$ to Eq.(2.16)-(2.20) are unique.

Proof. This follows directly from Theorem 2.2 and Lemma 2.7.

\section{Inverse Scattering}

The original point-source method reconstructs a time-harmonic wave satisfying the Sommerfeld radiation condition from its far field pattern. At the heart of the method is the construction of a backprojection operator with density $g$ such that the scattered field is calculated on some domain $\mathbb{E}$ by

$$
v^{s}(x, \kappa)=\int_{\mathbb{S}} e^{-i \kappa x \cdot \hat{y}} g(\hat{y}, \kappa) v^{\infty}(-\hat{y}, \kappa) d s(\hat{y}), \quad x \in \mathbb{E} .
$$


The backprojection operator relies on the duality of point-sources and plane waves. We denote the total field generated by an incident plane wave by $u$,

$$
u(x, \hat{\eta}, \kappa)=u^{i}(x, \hat{\eta}, \kappa)+u^{s}(x, \hat{\eta}, \kappa)
$$

where $u^{i}(x, \hat{\eta}, \kappa):=e^{i \kappa x \cdot \hat{\eta}}, \quad x \in \mathbb{R}^{m}, \kappa \in \mathbb{R}$, and $\hat{\eta} \in \mathbb{S}$ denotes the direction of incidence. The field resulting from excitation by a point-source $\Phi(x, z, \kappa)(x \neq z)$ is given by $w(\cdot, z, \kappa)$ : $\bar{\Omega}^{c} \rightarrow \mathbb{C}:$

$$
w(\cdot, z, \kappa):=w^{i}(\cdot, z, \kappa)+w^{s}(\cdot, z, \kappa),
$$

where $w^{i}(\cdot, z, \kappa):=\Phi(\cdot, z, \kappa), z \in \bar{\Omega}^{c}$, and $\kappa \neq 0$. The field $w$ is a solution to the scattering problem with an incident point-source. This field is the Green function for the boundary value problem Eq.(2.3), Eq.(2.4) (or Eq.(2.5) or Eq.(2.6)) and Eq.(2.7), and is symmetric: $w(x, z, \kappa)=w(z, x, \kappa) \quad x, z \in \bar{\Omega}^{c}, \quad x \neq z$. The corresponding scattered field $w^{s}(\cdot, z, \kappa)$ satisfies Eq.(2.3)-(2.7) with $f=-\Phi(\cdot, z, \kappa)$ on $\partial \Omega$.

\subsection{The time-dependent point-source method}

Consider $\Omega_{a} \subset \mathbb{R}^{m}$ a bounded domain with $C^{2}$ boundary. We first note that, for $\bar{\Omega} \subset \Omega_{a}$, a straight forward argument using Green's theorem and the boundary conditions for the fields $u$ and $w$ (see [10]) shows that

$$
w^{\infty}(-\hat{\eta}, z, \kappa)=\gamma(\kappa) u^{s}(z, \hat{\eta}, \kappa), \hat{\eta} \in \mathbb{S}, z \in \bar{\Omega}_{a}^{c},
$$

where

$$
\gamma(\kappa)= \begin{cases}\frac{e^{-i \frac{\pi}{4}}}{\sqrt{8 \pi \kappa}}, & m=2 \\ \frac{1}{4 \pi} & m=3\end{cases}
$$

Equation Eq.(3.4) is referred to as the mixed reciprocity relation and is discussed in further detail in [21, Theorem 2.1.4]. Second, by the principle of superposition for far field patterns [3, Theorem 3.16], the far field pattern due to scattering from any incident field (in particular an incident point-source) can be expressed as a superposition of far field patterns due to scattering from incident plane waves. The point-source method uses these two facts to reconstruct the scattered field on some region $\mathbb{E}$ outside of $\Omega$. This technique has been explored in [13-15,19-21] where it is applied to frequency-domain problems. Here we extend this methodology to nonharmonic, time-dependent waves.

Let $\Lambda \subset \mathbb{S}$ denote an open set of directions on $\mathbb{S}$. Here, $\Lambda$ models the aperture on which our sensors lie. In our numerical experiments, this is a symmetric interval of the unit sphere centered with respect to the direction of the incident field. The far field $u^{\infty}$ due to an incident plane wave with direction $\hat{\eta} \in \mathbb{S}$ is measured at points $\hat{y} \in \Lambda$. Define the Herglotz wave operator $H_{\kappa}: L^{2}(-\Lambda) \rightarrow L^{\infty}\left(\mathbb{R}^{m}\right)$ by

$$
\left(H_{\kappa} g\right)(x):=\int_{\Lambda} e^{i \kappa x \cdot(-\hat{y})} g(-\hat{y}) d s(\hat{y}), \quad x \in \mathbb{R}^{m}, g \in L^{2}(-\Lambda) .
$$


The corresponding family of Herglotz wave functions parameterized by $\kappa$ and mapping $\mathbb{R}^{m}$ to $\mathbb{C}, h_{g}(\cdot, \kappa):=\left(H_{\kappa} g\right)(\cdot)$, consists of entire solutions to the Helmholtz equation for fixed $\kappa$. Of particular interest is the Herglotz wave operator restricted to some surface $\mathbb{X} \subset \mathbb{R}^{m}$. The adjoint of $H_{\kappa}$ with $\kappa$ fixed, denoted $H_{\kappa}^{*}: L^{2}(\mathbb{X}) \rightarrow L^{2}(\Lambda)$, is given by

$$
\left(H_{\kappa}^{*} \psi\right)(\hat{\eta}):=\int_{\mathbb{X}} e^{-i \kappa x \cdot(-\hat{\eta})} \psi(x) d s(x), \quad \hat{\eta} \in \Lambda .
$$

Let $\Omega_{a}$ be a bounded domain with simply connected, $C^{2}$ boundary and $\Omega \subset \operatorname{int} \Omega_{a}$. Suppose $\kappa \notin \mathcal{K}$ where

$$
\mathcal{K} \equiv\left\{\kappa \in \mathbb{R} \mid \kappa^{2} \text { is an eigenvalue of }-\triangle \text { on } \Omega_{a}\right\} .
$$

It can then be shown that $H_{\kappa}$ and $H_{\kappa}^{*}$ restricted to $\partial \Omega_{a}$ are injective with dense range. Thus one can choose the Herglotz wave function $h_{g}$ with density $g$ to approximate arbitrarily closely any convenient incident field $v^{i}$ on $\partial \Omega_{a}$. The incident field we approximate is an incident point-source located at a point $z \in \bar{\Omega}_{a}^{c}$. To see this, define the Herglotz wave function as a function of the spatial variable $x \in \Omega_{a}$ and wavenumber $\kappa \in \mathbb{R}$ parameterized by the point $z$ as

$$
h_{g}(x, z, \kappa):=\int_{\Lambda} e^{i \kappa x \cdot(-\hat{y})} g(-\hat{y}, z, \kappa) d s(\hat{y}) .
$$

The backprojection operator that is central to the point-source method is built upon the integral operator $\mathcal{B}_{g}: L^{2}(\Lambda \times \mathbb{S} \times \mathbb{R}) \rightarrow L^{2}\left(\mathbb{R}^{m} \times \mathbb{S} \times \mathbb{R}\right)$ with kernel $g(\cdot, z, \cdot)$. For a function $\psi \in L^{2}(\Lambda \times \mathbb{S} \times \mathbb{R})$, the operator $\mathcal{B}_{g}$ is defined by

$$
\left(\mathcal{B}_{g} \psi\right)(z, \hat{\eta}, \kappa):=\int_{\Lambda} \psi(\hat{y}, \hat{\eta}, \kappa) \frac{g(-\hat{y}, z, \kappa)}{\gamma(\kappa)} d s(\hat{y}),
$$

for $\gamma(\kappa)$ given by Eq.(3.5). The corresponding time-domain operator, denoted $B_{g}: L^{2}(\Lambda \times$ $\mathbb{S} \times \mathbb{R}) \rightarrow L^{2}\left(\mathbb{R}^{m} \times \mathbb{S} \times \mathbb{R}\right)$, is defined by

$$
B_{g}:=\mathcal{F}_{\kappa} \mathcal{B}_{g} \mathcal{F}_{t}
$$

Remark 3.1 The dependency of $g$ on $\kappa$ is essential to the convergence properties of reconstructions in the time domain. For technical reasons mentioned in Remark 2.1, in two dimensions we exclude the zero frequency. In Lemma 3.6 and Theorem 3.7 below, we also restrict ourselves to the cases where the incoming wave is compactly supported with respect to $\kappa$ on $\mathbb{R} \backslash\{0\}$ in order to assure the regularity of the inversion process in the time domain. Again, this is not physically unreasonable since sources with unbounded bandwidth are indeed rare.

Theorem 3.3 states that, provided $\Omega \subset \Omega_{a}$, the density $g(\cdot, z, \kappa)$ for which the Herglotz wave function approximates $\Phi(\cdot, z, \kappa)$ on $\partial \Omega_{a}$ allows one directly to calculate an approximation to the scattered field $u^{s}$ at the point $z$ from fixed frequency far field data $u^{\infty}$. For multifrequency fields in the time-domain, we are interested in the uniformity of such approximations with respect to $\kappa$. For this we need the following lemma. 
Lemma 3.2 Let $\Omega_{a} \subset \mathbb{R}^{m}$ be a bounded domain with simply connected, $C^{2}$ boundary. The set of Dirichlet eigenvalues of the negative Laplacian on the interior of $\Omega_{a}$ has measure zero with respect to Lebesgue measure on $\mathbb{R}$.

Proof. The spectrum of $-\triangle$ on bounded domains has a countably infinite spectrum [12, Theorem 4.1], and any countable set of points in $\mathbb{R}$ has measure zero with respect to Lebesgue measure.

Before stating the main result of this section, we introduce the far field mapping $\mathcal{T}_{\Omega_{a}^{\prime}}$ : $\left.v^{s}\right|_{\Omega_{a}^{\prime}} \rightarrow v^{\infty}$ mapping the scattered field $v^{s}$ restricted to any compact subset $\Omega_{a}^{\prime} \subset \bar{\Omega}^{c}$ containing open subsets to the far field $v^{\infty}$. This is a continuous mapping. The time domain counterpart to this - also a continuous mapping - is denoted $T$ with $\mathcal{T}_{\Omega_{a}^{\prime}}:\left.v^{s}\right|_{\Omega_{a}^{\prime}} \rightarrow v^{\infty}$. The next theorems show how to approximate the inverse of the far field mapping of the scattered field due to an incident plane wave.

Theorem 3.3 (Norm convergence in frequency) Let $\Omega_{a} \subset \mathbb{R}^{m}$ be a bounded domain with simply connected, $C^{2}$ boundary satisfying $\bar{\Omega} \subset \Omega_{a}$ and let $\mathbb{K}=\mathbb{R} \backslash \mathbb{B}\left(0, \epsilon^{\prime}\right)$ where $\mathbb{B}\left(0, \epsilon^{\prime}\right)$ is the closed ball of radius $\epsilon^{\prime}$ centered at the origin. Given any $\delta>0$ and any fixed $z \in \bar{\Omega}_{a}^{c}$, there exists an $\epsilon>0$ such that, for all $\hat{\eta} \in \mathbb{S}$, and g satisfying

$$
\left\|\Phi(\cdot, z, \cdot)-h_{g}(\cdot, z, \cdot)\right\|_{C\left(\partial \Omega_{a}\right) \times L^{2}(\mathbb{K})}^{2}<\epsilon
$$

we have

$$
\left\|u^{s}(z, \hat{\eta}, \cdot)-\left(\mathcal{B}_{g} u^{\infty}\right)(z, \hat{\eta}, \cdot)\right\|_{L^{2}(\mathbb{K})}^{2}<\delta .
$$

Here $u^{s}$ and $u^{\infty}$ are the scattered field and far field pattern due to an incident plane wave with direction $\hat{\eta}, \mathcal{B}_{g}$ is defined by Eq.(3.9), and $h_{g}(\cdot, z, \cdot)$ is defined by Eq.(3.8).

Proof. The Herglotz wave operator $H_{\kappa}$ is injective with dense range on $\partial \Omega_{a}$ for all $\kappa \in \mathbb{K} \backslash \mathcal{K}$ where $\mathcal{K}$ is defined by Eq.(3.7). Recall (Lemma 3.2) that $\mathcal{K}$ is countable, so we will index elements of this set by $\kappa_{j}$. Since special care is needed for the frequencies in $\mathcal{K}$, we exclude small neighborhoods of these frequencies. More precisely, let $\mathbb{K}^{\prime} \equiv \cup_{\kappa_{j} \in \mathcal{K}} \mathbb{B}\left(\kappa_{j}, \epsilon_{j}^{\prime}\right)$. We then split the norm with respect to $\kappa$ in Eq.(3.11) into two parts:

$$
\begin{aligned}
\left\|\Phi(\cdot, z, \cdot)-h_{g}(\cdot, z, \cdot)\right\|_{C\left(\partial \Omega_{a}\right) \times L^{2}(\mathbb{K})}^{2}= & \left\|\Phi(\cdot, z, \cdot)-h_{g}(\cdot, z, \cdot)\right\|_{C\left(\partial \Omega_{a}\right) \times L^{2}\left(\mathbb{K} \backslash \mathbb{K}^{\prime}\right)}^{2} \\
& +\left\|\Phi(\cdot, z, \cdot)-h_{g}(\cdot, z, \cdot)\right\|_{C\left(\partial \Omega_{a}\right) \times L^{2}\left(\mathbb{K}^{\prime}\right)}^{2} .
\end{aligned}
$$

It follows immediately from the denseness of the Herglotz wave operator on $\mathbb{K} \backslash \mathbb{K}^{\prime}$ that for all $\epsilon>0$ there is a density $g$ such that

$$
\left\|\Phi(\cdot, z, \cdot)-h_{g}(\cdot, z, \cdot)\right\|_{C\left(\partial \Omega_{a}\right) \times L^{2}\left(\mathbb{K} \backslash \mathbb{K}^{\prime}\right)}^{2} \leq \frac{\epsilon}{2} .
$$


The corresponding Herglotz wave function $h_{g}$ and $\Phi$ solve Eq.(2.3) for each fixed $\kappa \in \mathbb{K} \backslash \mathbb{K}^{\prime}$ on the interior of $\Omega_{a}$ and thus are analytic with respect to the spatial variable [3, Theorem 2.2] and the bound in Eq.(3.11) extends to the interior of $\Omega_{a}$, that is, for some $c^{\prime}>0$

$$
\left\|\Phi(\cdot, z, \cdot)-h_{g}(\cdot, z, \cdot)\right\|_{C\left(\bar{\Omega}_{a}\right) \times L^{2}\left(\mathbb{K} \backslash \mathbb{K}^{\prime}\right)}^{2} \leq c^{\prime} \frac{\epsilon}{2} .
$$

On $\mathbb{K}^{\prime}$ we fix any $\bar{\kappa}_{j} \in \mathbb{B}\left(\kappa_{j}, \epsilon_{j}^{\prime}\right) \backslash\left\{\kappa_{j}\right\}$ and construct a constant density $\bar{g}_{j}(\hat{y}, z, \cdot)$ on $\mathbb{B}\left(\epsilon_{j}^{\prime}, \kappa_{j}\right)$ that satisfies

$$
\left\|\Phi\left(\cdot, z, \bar{\kappa}_{j}\right)-h_{\bar{g}_{j}}\left(\cdot, z, \bar{\kappa}_{j}\right)\right\|_{C\left(\partial \Omega_{a}\right)}^{2} \leq \epsilon_{j}^{\prime \prime}
$$

We show below that, for $\epsilon_{j}^{\prime}$ and $\epsilon_{j}^{\prime \prime}$ small enough, these constant densities on $\mathbb{B}\left(\kappa_{j}, \epsilon_{j}^{\prime}\right)$ satisfy

$$
\left\|\Phi(\cdot, z, \cdot)-h_{\bar{g}}(\cdot, z, \cdot)\right\|_{C\left(\partial \Omega_{a}\right) \times L^{2}\left(\mathbb{K}^{\prime}\right)}^{2} \leq \frac{\epsilon}{2} .
$$

Moreover, by our construction, this bound can be carried into the interior of $\Omega_{a}$ to yield, for some constant $c^{\prime}>0$,

$$
\left\|\Phi(\cdot, z, \cdot)-h_{\bar{g}}(\cdot, z, \cdot)\right\|_{C\left(\bar{\Omega}_{a}\right) \times L^{2}\left(\mathbb{K}^{\prime}\right)}^{2} \leq c^{\prime} \frac{\epsilon}{2}
$$

For the moment, we take this for granted.

Equations (3.14) and (3.17) together yield

$$
\left\|\Phi(\cdot, z, \cdot)-h_{g}(\cdot, z, \cdot)\right\|_{C(\partial \Omega) \times L^{2}(\mathbb{K})}^{2} \leq c \epsilon
$$

for some constant $c>0$. Now consider the scattered field associated with the incident field $v^{i}(x, z, \kappa)=h_{g}(x, z, \kappa)$ due to scattering from the sound-soft obstacle $\Omega$. Likewise, consider the scattered field $w^{s}$ due to an incident point source $\Phi(x, z, \kappa), z \in \Omega_{a}^{c}$. On $\partial \Omega$ we have $v^{i}=-v^{s}$ and $w^{s}=-\Phi$, thus Eq.(3.18) can be rewritten as

$$
\left\|-w^{s}(\cdot, z, \cdot)+v^{s}(\cdot, z, \cdot)\right\|_{C(\partial \Omega) \times L^{2}(\mathbb{K})}^{2} \leq c \epsilon .
$$

Since the far field mapping is $\mathcal{T}_{\partial \Omega}$ is a continuous mapping, for any choice of $\delta>0$ there is an $\epsilon>0$ such that $\left\|w^{s}(\cdot, z, \cdot)-v^{s}(\cdot, z, \cdot)\right\|_{C(\partial \Omega) \times L^{2}(\mathbb{K})}^{2} \leq c \epsilon$ implies

$$
\left\|w^{\infty}(-\hat{\eta}, z, \cdot)-v^{\infty}(-\hat{\eta}, z, \cdot)\right\|_{L^{2}(\mathbb{K})}^{2} \leq \delta, \quad \forall \hat{\eta} \in \mathbb{S} .
$$

By [3, Lemma 3.16], $v^{\infty}(-\hat{\eta}, z, \kappa)=\int_{\Lambda} u^{\infty}(-\hat{\eta},-\hat{y}, \kappa) g(-\hat{y}, z, \kappa) d s(\hat{y})$, where $u^{\infty}$ is the far field pattern due to scattering of an incident plane wave. Now, the mixed reciprocity relation 
Eq.(3.4) together with the standard reciprocity relation $u^{\infty}(-\hat{\eta},-\hat{y}, \kappa)=u^{\infty}(\hat{y}, \hat{\eta}, \kappa)$ (see $[3$, Theorem 3.13]) yield the result

$$
\left\|u^{s}(z, \hat{\eta}, \cdot)-\frac{1}{\gamma(\kappa)} \int_{\Lambda} u^{\infty}(\hat{y}, \hat{\eta}, \cdot) g(-\hat{y}, z, \cdot) d s(\hat{y})\right\|_{L^{2}(\mathbb{K})}^{2} \leq \delta, \quad \forall \hat{\eta} \in \mathbb{S} .
$$

What remains is to show Eq.(3.16) and (3.17). For each $j$ and a given $\epsilon_{j}^{\prime}$ and $\epsilon_{j}^{\prime \prime}$, choose any $\bar{\kappa}_{j} \in \mathbb{B}\left(\kappa_{j}, \epsilon_{j}^{\prime}\right) \backslash\left\{\kappa_{j}\right\}$ and let $\bar{g}_{j}$ satisfy Eq.(3.15) (again, possible because at $\bar{\kappa}_{j}$ the Herglotz wave operator is injective with dense range on $C\left(\partial \Omega_{a}\right)$ ). For this choice of $\bar{\kappa}_{j}$, the bound in Eq.(3.15) can be extended to the interior, that is, there is a constant $c_{j}^{\prime}$ such that

$$
\left\|\Phi\left(\cdot, z, \bar{\kappa}_{j}\right)-h_{\bar{g}_{j}}\left(\cdot, z, \bar{\kappa}_{j}\right)\right\|_{C\left(\bar{\Omega}_{a}\right)}^{2} \leq c_{j}^{\prime} \epsilon_{j}^{\prime \prime} .
$$

Define

$$
\rho\left(\epsilon_{j}^{\prime}, \kappa_{j}\right) \equiv \max _{\kappa \in \overline{\mathbb{B}}\left(\kappa_{j}, \epsilon_{j}^{\prime}\right)}\left\|\Phi(\cdot, z, \kappa)-\Phi\left(\cdot, z, \bar{\kappa}_{j}\right)\right\|_{C\left(\partial \Omega_{a}\right)}^{2}+\left\|h_{\bar{g}_{j}}\left(\cdot, z, \bar{\kappa}_{j}\right)-h_{\bar{g}_{j}}(\cdot, z, \kappa)\right\|_{C\left(\partial \Omega_{a}\right)}^{2}
$$

where $\overline{\mathbb{B}}$ is the closure of the ball. We write "max" instead of "sup" to emphasize that the supremum is attained since the feasible set is compact and the objective is continuous on this compact set; moreover $\rho$ is a continuous function of $\epsilon_{j}^{\prime}>0$ with $\rho\left(\epsilon_{j}^{\prime}, \kappa_{j}\right) \rightarrow 0$ as $\epsilon_{j}^{\prime} \rightarrow 0$. This bound can also be continued to the interior of $\Omega_{a}$ because the objective, which we know explicitly, is a continuous (in fact, analytic) function of both the spatial variable and $\kappa$ on $\bar{\Omega}_{a} \times \overline{\mathbb{B}}\left(\kappa_{j}, \epsilon_{j}^{\prime}\right)$, that is,

$$
\max _{\kappa \in \overline{\mathbb{B}}\left(\kappa_{j}, \epsilon_{j}^{\prime}\right)}\left\|\Phi(\cdot, z, \kappa)-\Phi\left(\cdot, z, \bar{\kappa}_{j}\right)\right\|_{C\left(\bar{\Omega}_{a}\right)}^{2}+\left\|h_{\bar{g}_{j}}\left(\cdot, z, \bar{\kappa}_{j}\right)-h_{\bar{g}_{j}}(\cdot, z, \kappa)\right\|_{C\left(\bar{\Omega}_{a}\right)}^{2} \leq c_{j}^{\prime} \rho\left(\epsilon_{j}^{\prime}, \kappa_{j}\right)
$$

Together Eq.(3.19) and Eq.(3.21) yield, for all $\kappa \in \mathbb{B}\left(\kappa_{j}, \epsilon_{j}^{\prime}\right)$ and some $c_{j}^{\prime}>0$,

$$
\begin{aligned}
\left\|\Phi(\cdot, z, \kappa)-h_{\bar{g}_{j}}(\cdot, z, \kappa)\right\|_{C\left(\bar{\Omega}_{a}\right)}^{2} \leq & \left\|\Phi(\cdot, z, \kappa)-\Phi\left(\cdot, z, \bar{\kappa}_{j}\right)\right\|_{C\left(\bar{\Omega}_{a}\right)}^{2} \\
& +\left\|h_{\bar{g}_{j}}\left(\cdot, z, \bar{\kappa}_{j}\right)-h_{\bar{g}_{j}}(\cdot, z, \kappa)\right\|_{C\left(\bar{\Omega}_{a}\right)}^{2} \\
& +\left\|\Phi\left(\cdot, z, \bar{\kappa}_{j}\right)-h_{\bar{g}_{j}}\left(\cdot, z, \bar{\kappa}_{j}\right)\right\|_{C\left(\bar{\Omega}_{a}\right)}^{2} \\
\leq & c_{j}^{\prime}\left(\rho\left(\epsilon_{j}^{\prime}, \kappa_{j}\right)+\epsilon_{j}^{\prime \prime}\right)
\end{aligned}
$$

The pointwise - with respect to $\kappa$ - bound of Eq.(3.22) then yields

$$
\begin{aligned}
\| \Phi(\cdot, z, \cdot)-h_{\bar{g}}\left(\cdot, z, \cdot \|_{C\left(\bar{\Omega}_{a}\right) \times L^{2}\left(\mathbb{K}^{\prime}\right)}^{2}\right. & =\int_{\mathbb{K}^{\prime}}\left\|\Phi(\cdot, z, \kappa)-h_{g}(\cdot, z, \kappa)\right\|_{C\left(\bar{\Omega}_{a}\right)}^{2} d \kappa \\
& \leq \sum_{j} 2 c_{j}^{\prime} \epsilon_{j}^{\prime}\left(\rho\left(\epsilon_{j}^{\prime}, \kappa_{j}\right)+\epsilon_{j}^{\prime \prime}\right) .
\end{aligned}
$$


Since the neighborhood radius $\epsilon_{j}^{\prime}$ and approximation error $\epsilon_{j}^{\prime \prime}$ are arbitrary, they can be chosen so that

$$
\sum_{j} 2 c_{j}^{\prime} \epsilon_{j}\left(\rho\left(\epsilon_{j}^{\prime}, \kappa_{j}\right)+\epsilon_{j}^{\prime \prime}\right)<c^{\prime} \frac{\epsilon}{2} .
$$

which establishes Eq.(3.17)-(3.17) and completes the proof.

Corollary 3.4 (Pointwise convergence in time) In addition to the assumptions of Theorem 3.3, let $u^{i}(\cdot, \hat{\eta}, \kappa)=0$ for all $\kappa \in \mathbb{B}\left(0, \epsilon^{\prime}\right)$ then there exists a sequence $\left\{g_{j}\right\}$ such that

$$
\left(B_{g_{j}} U^{\infty}\right)(z, \hat{\eta}, t) \rightarrow U^{s}(z, \hat{\eta}, t), \quad \forall \hat{\eta} \in \mathbb{S},
$$

Proof. The region $\mathbb{K}$ is not assumed to be compact, so the usual justification does not apply. Instead, note that, since $u^{i}(\cdot, \kappa, \hat{\eta})=0$ for $\kappa \in \mathbb{B}\left(0, \epsilon^{\prime}\right)$, the scattered field and far field pattern at these wavenumbers are also zero. Without loss of generality define $g(\hat{y}, z, \kappa):=0$ for $\kappa \in \mathbb{B}\left(0, \epsilon^{\prime}\right)$. Thus, the inequality Eq.(3.12) can be extended to

$$
\left\|u^{s}(z, \hat{\eta}, \cdot)-\left(\mathcal{B}_{g} u^{\infty}\right)(z, \hat{\eta}, \cdot)\right\|_{L^{2}(\mathbb{R})}<\delta .
$$

By Parseval's identity, we have

$$
\left\|U^{s}(z, \hat{\eta}, \cdot)-\left(B_{g} U^{\infty}\right)(z, \hat{\eta}, \cdot)\right\|_{L^{2}(\mathbb{R})}<\delta
$$

where $U^{s}=\mathcal{F}_{\kappa} u^{s}, U^{\infty}=\mathcal{F}_{\kappa} u^{\infty}$ and $B$ is defined by Eq.(3.10). It follows from [24, Theorem 3.12 ] that there exists a sequence $\left\{g_{j}\right\}$ - which can be constructed as a subsequence of another sequence of densities for which the bound in Eq.(3.11) converges to zero - such that

$$
\left(B_{g_{j}} U^{\infty}\right)(z, \hat{\eta}, t) \rightarrow U^{s}(z, \hat{\eta}, t), \quad \forall \hat{\eta} \in \mathbb{S} \text {, and a.e. } t,
$$

where $B_{g_{i_{j}}}$ is defined by Eq.(3.10). Moreover, since $U^{s}(z, \hat{\eta}, \cdot)$ and $\left(B_{g_{i}} U^{\infty}\right)(z, \hat{\eta}, \cdot) \in C^{2}(\mathbb{R})$ convergence is pointwise everywhere.

We close this section with a few remarks about the regularity of the point-source method, by which we mean that the method admits a regular regularization strategy. Noisy inverse problems are characterized by a mismatch, or error, between the true image $\psi$ of the input $\varphi$ under the operator $A, \psi=A \varphi$, and the observed image $\psi_{\delta}$. The next definition establishes the framework for analyzing the convergence properties of regularized inversion in the presence of noise.

Definition 3.5 $A$ regularization strategy $R_{\alpha}$, that is a rule for choosing $\alpha$ depending on the size of the image error $\delta$, is called regular if, for all $\psi \in$ range $A$ and all $\psi_{\delta} \in \mathbb{Y}$ with $\left\|\psi_{\delta}-\psi\right\| \leq \delta$ we have

$$
R_{\alpha(\delta)} \psi_{\delta} \rightarrow A^{-1} \psi, \text { as } \delta \rightarrow 0
$$


Lemma 3.6 The density

$$
g_{\alpha}(\cdot, z, \kappa):=\left(H_{\kappa}^{*} H_{\kappa}+\alpha I\right)^{-1} H_{\kappa}^{*} \Phi(\cdot, z, \kappa)
$$

depends continuously on $\kappa \in \mathbb{R} \backslash\{0\}$. The norm $\left\|g_{\alpha}(\cdot, z, \kappa)\right\|_{L^{2}(\Lambda)}$ is uniformly bounded with respect to $\kappa$ on compact subsets of $\mathbb{R} \backslash\{0\}$.

PRoOF. First, we remark that the Tikhonov operator $\left(H_{\kappa}^{*} H_{\kappa}+\alpha I\right)^{-1} H_{\kappa}^{*}$ is well defined for all $\kappa \in \mathbb{R}$. The operator $H_{\alpha}$ depends continuously on $\kappa$ for all $\kappa \in \mathbb{R}$, since its kernel is analytic in $\kappa$. Also, the function $\Phi$ depends continuously on $\kappa$ for $\kappa \in \mathbb{R} \backslash\{0\}$. But then by standard arguments the solution $g_{\alpha}$ depends continuously on $\kappa \in \mathbb{R} \backslash\{0\}$ in its $L^{2}$-norm on $\Lambda$. A continuous function is bounded on compact subsets of its domain, this yields the second statement.

Theorem 3.7 (Regularity of the time-domain point-source method) The density $g_{\alpha}(\hat{y}, z, \kappa)$ given by (3.26) is a regularized solution (in fact, the Tikhonov regularized solution) to the inverse problem

$$
\left(H_{\kappa} g\right)(x, z, \kappa)=\Phi(x, z, \kappa), \quad x \in \partial \Omega_{a}
$$

for $\kappa \in \mathbb{K} \subset \mathbb{R} \backslash\{0\}$ compact. Moreover, under the assumptions of Corollary 3.4, the operator $B_{g_{\alpha}}$ defined by Eq.(3.10) is a regularized inverse of the far field mapping $T_{\Omega_{a}^{\prime}}$ on compact subsets of $\bar{\Omega}^{c}$, which, in the presence of noise, admits a regular regularization strategy.

Proof. Recall that $H_{\kappa}$ and $H_{\kappa}^{*}$, restricted to $\partial \Omega_{a}$ for fixed $\kappa$ and $\kappa^{2}$ not a Dirichlet eigenvalue of $-\triangle$ on $\Omega_{a}$, are injective with dense range. Thus the operator $R_{\alpha}=$ $\left(\alpha I+H_{\kappa}^{*} H_{\kappa}\right)^{-1} H_{\kappa}^{*}$ is the Tikhonov regularized inverse operator for $H_{\kappa}$ on $\partial \Omega_{a}$, for $\kappa \in$ $\mathbb{K} \backslash\left\{\right.$ Dirichlet eigenvalues of $-\triangle$ on $\left.\partial \Omega_{a}\right\}$ (see for example [3, Ch.4]).

To prove the remainder of the theorem we decompose the error into the regularization error and the data error:

$$
\left\|B_{g_{\alpha}} U_{\delta^{\prime}}^{\infty}-T_{\Omega_{a}^{\prime}}^{-1} U^{\infty}\right\| \leq\left\|B_{g_{\alpha}} U^{\infty}-T_{\Omega_{a}^{\prime}}^{-1} U^{\infty}\right\|+\left\|B_{g_{\alpha}}\left(U^{\infty}-U_{\delta^{\prime}}^{\infty}\right)\right\|
$$

where $U_{\delta^{\prime}}^{\infty}$ is a noisy measurement satisfying $\left\|U^{\infty}(z, \hat{\eta}, t)-U_{\delta^{\prime}}^{\infty}(z, \hat{\eta}, t)\right\|<\delta^{\prime}(t)$. To show that $B_{g_{\alpha}}$ is a regularized inverse of $T_{\Omega_{a}^{\prime}}$, we need only show that, for all $U^{\infty} \in$ range $T_{\Omega_{a}^{\prime}}$, the first norm on the right of Eq.(3.27) tends to zero as $\alpha \rightarrow 0$. If the second norm on the right of Eq.(3.27) tends to zero as the data error $\left\|U^{\infty}(z, \hat{\eta}, t)-U_{\delta^{\prime}}^{\infty}(z, \hat{\eta}, t)\right\|<\delta^{\prime}(t) \rightarrow 0$, then $B_{g_{\alpha}}$ is regular.

First, assuming that $u^{s}(\cdot, \hat{\eta}, \kappa)=0$ for all $\kappa \in \mathbb{K}^{c}$, then by Eq.(3.25), given any $\delta>0$ and any $U^{\infty} \in$ range $T_{\{z\}}$, there exists $\epsilon>0$ and $\alpha>0$ such that

$$
\left\|\Phi(x, z, \kappa)-\left(H_{\kappa} g_{\alpha}\right)(x, z, \kappa)\right\|_{C\left(\partial \Omega_{a}\right) \times L^{2}(\mathbb{K})}<\epsilon
$$

implies

$$
\left\|\left(T_{\{z\}}^{-1} U^{\infty}\right)(z, \hat{\eta}, \cdot)-\left(B_{g_{\alpha}} U^{\infty}\right)(z, \hat{\eta}, \cdot)\right\|_{L^{2}(\mathbb{R})}<\frac{\delta}{2}, \quad \forall \hat{\eta} \in \mathbb{S} .
$$


Since $\left(\alpha I+H_{\kappa}^{*} H_{\kappa}\right)^{-1} H_{\kappa}^{*}$ is a regularized inverse of $H_{\kappa}$, which is injective with dense range, the norm above tends to zero as $\epsilon$, and $\alpha \rightarrow 0$.

For the second step, recall the definition $B_{g_{\alpha}}=\mathcal{F}_{\kappa} \mathcal{B}_{g} \mathcal{F}_{t}$. The function

$$
\mathcal{F}_{t}\left(U^{\infty}(z, \hat{\eta}, \cdot)-U_{\delta}^{\infty}(z, \hat{\eta}, \cdot)\right)
$$

has compact support $\mathbb{K}$ with respect to $\kappa$. On $\mathbb{K}$ the operator $g_{\alpha}$ is uniformly bounded and thus also the backprojection operators $\mathcal{B}_{g}$ are bounded as operators $L^{2}(\Lambda)$ into the set of continuous functions for any appropriate compact subset $M$ of the open exterior of the scatterer $\Omega$ uniformly for $\kappa \in \mathbb{K}$. Since $\mathcal{B}_{g}$ are pointwise in frequency and uniformly bounded on $\mathbb{K}$, for given $\delta / 2>0$ there is $\delta^{\prime}$ such that

$$
\left\|U^{\infty}(z, \hat{\eta}, \cdot)-U_{\delta^{\prime}}^{\infty}(z, \hat{\eta}, \cdot)\right\|_{L^{2}(\mathbb{R})} \leq \delta^{\prime}
$$

yields

$$
\left\|\mathcal{F}_{\kappa} \mathcal{B}_{g} \mathcal{F}_{t}\left(U^{\infty}(z, \hat{\eta}, \cdot)-U_{\delta^{\prime}}^{\infty}(z, \hat{\eta}, \cdot)\right)\right\|_{L^{2}(\mathbb{R})} \leq \frac{\delta}{2}, \quad z \in M
$$

This yields

$$
\left\|B_{g_{\alpha}} U^{\infty}(z, \hat{\eta}, \cdot)-B_{g_{\alpha}} U_{\delta^{\prime}}^{\infty}(z, \hat{\eta}, \cdot)\right\|_{L^{2}(\mathbb{R})} \leq \frac{\delta}{2}, \quad \forall \hat{\eta} \in \mathbb{S}, z \in M .
$$

Together Eq.(3.28) and Eq.(3.32) yield

$$
\left\|T_{\{z\}}^{-1} U^{\infty}(z, \hat{\eta}, \cdot)-B_{g_{\alpha}} U_{\delta^{\prime}}^{\infty}(z, \hat{\eta}, \cdot)\right\|_{L^{2}(\mathbb{R})}<\delta, \quad \forall \hat{\eta} \in \mathbb{S}, z \in M,
$$

and thus the regularity of the point-source method.

Remark 3.8 Theorem 3.7 relies on some strong, but practical, assumptions about the nature of the noise. Specifically, we assume that the error in the time dependent far field pattern $U_{\delta^{\prime}}^{\infty}$ is band-limited and it is integrable with respect to time.

\section{Numerical examples}

We conclude with two numerical demonstrations that illustrate the advantage of the time domain formulation over straight averaging over multi-frequency data. We begin with a few remarks about a realization of the method for discretely sampled data.

Again, the set of values of $\mathcal{K}$ defined by Eq.(3.7) is countable. Given $\epsilon>0$ we choose a discretization $\bar{\kappa}_{0}<\bar{\kappa}_{1}<\ldots<\bar{\kappa}_{j}<\ldots \bar{\kappa}_{n} \in \mathbb{K} \backslash \mathcal{K}$ such that for every $\kappa \in \mathbb{K}$ there is $j \in\{1, \ldots, n\}$ such that

$$
\left\|\Phi(\cdot, z, \kappa)-\Phi\left(\cdot, z, \bar{\kappa}_{j}\right)\right\|_{C\left(\partial \Omega_{a}\right)}^{2} \leq \frac{\epsilon}{3 n}
$$


uniformly on $\mathbb{K}$. We construct the density $g_{\alpha, j}\left(\cdot, z, \bar{\kappa}_{j}\right)$ via Eq.(3.26) for $H_{\bar{\kappa}_{j}}$ and its adjoint restricted to $\partial \Omega_{a}$ where $\alpha$ is chosen so that

$$
\left\|\Phi\left(\cdot, z, \bar{\kappa}_{j}\right)-h_{g_{\alpha, j}}\left(\cdot, z, \bar{\kappa}_{j}\right)\right\|_{C\left(\partial \Omega_{a}\right)} \leq \frac{\epsilon}{3 n}
$$

and

$$
\left\|h_{g_{\alpha, j}}\left(\cdot, z, \bar{\kappa}_{j}\right)-h_{g_{\alpha, j}}(\cdot, z, \kappa)\right\|_{C\left(\partial \Omega_{a}\right)}^{2} \leq \frac{\epsilon}{3 n}
$$

for all $j=0, \ldots, n$ and all nearest $\kappa \in \mathbb{K}$. We define the density on the continuum of wavenumbers, $g_{\alpha}(\cdot, z, \kappa)$, by piecewise constant interpolation over the wavenumber grid, that is, $g_{\alpha}(\cdot, z, \kappa)=g_{\alpha, j}\left(\cdot, z, \bar{\kappa}_{j}\right)$ where $\bar{\kappa}_{j}$ is the nearest grid point to a given $\kappa$. Similar to Eq.(3.22), this leads to the estimate for each $j$

$$
\begin{aligned}
\left\|\Phi(\cdot, z, \kappa)-h_{g_{\alpha}}(\cdot, z, \kappa)\right\|_{C\left(\partial \Omega_{a}\right) \leq} & \left\|\Phi(\cdot, z, \kappa)-\Phi\left(\cdot, z, \bar{\kappa}_{j}\right)\right\|_{C\left(\partial \Omega_{a}\right)} \\
& +\| h_{g_{\alpha, j}\left(\cdot, z, \bar{\kappa}_{j}\right)-h_{g_{\alpha, j}}(\cdot, z, \kappa) \|_{C\left(\partial \Omega_{a}\right)}^{2}} \\
& +\| \Phi\left(\cdot, z, \bar{\kappa}_{j}\right)-h_{g_{\alpha, j}\left(\cdot, z, \bar{\kappa}_{j}\right) \|_{C\left(\partial \Omega_{a}\right)}^{2}} \\
\leq &
\end{aligned}
$$

where $\bar{\kappa}_{j}$ is the nearest discretization point to $\kappa$.

In Figure 3 we show the actual and reconstructed time progression of the total wave-field (scattered plus incident waves) as it scatters around a sound soft obstacle. The incident wave travels from right to left, and has the frequency profile shown in Figure 1. As this picture shows, the incident field, that is, the boundary condition $f$ in Eq.(2.4), has been constructed so that it satisfies the hypotheses of Lemma 2.7. The obstacle is the well known kite shown in Figure 2. Note that the wave numbers range from -5 to 5 and the obstacle diameters are around 2, thus the wave numbers are in the resonance region of the obstacles.

The data consists of time-series measurements of the scattered field on a sphere $\mathbb{S}$ in the far field. To generate the forward data we use the integral equation techniques described in [3]. We discretize the integral equations using 160 points on the boundary, ignoring the singularity. We evaluate the far field pattern at 80 points on the unit sphere with 66 time slices for two different incident field directions. The scattered field, reconstructed using the point-source method, was computed at 6400 evenly spaced radial points in the computational domain. For reference, we calculate the true scattered field via boundary integral techniques on a $80 \times 80$ Cartesian grid.

To implement the point-source method in this setting, we apply the Fourier transform to the far field data and solve for the scattered field at the sampled wavenumber $\kappa$ in the usual way with the point-source method (see [19,21]). We refine our solution for the scattered fields at each frequency by estimating the location of the center of the obstacle via a first-order physical optics approximation for the backprojection density as described in [14]. With the location of the center in hand, we then reconstruct the total field at each frequency along radial lines extending from this center. Applying the inverse Fourier transform yields the 


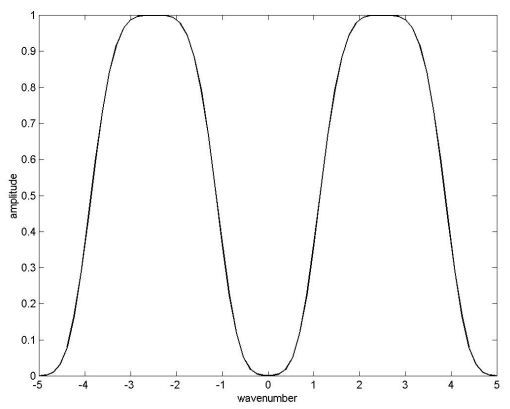

Figure 1: Frequency profile of incident wave.

time-dependent wave. This is shown in the series of snap-shots of the wave displayed in Figure 3.

The advantage of imaging with pulses is demonstrated in Figure 4. In the first image, Figure 4(a), we compute the time-average (66 time samples) of the total field amplitude over the two incident pulses. In the second image, Figure 4(b), we compute the frequency-average (66 frequencies) of the total field amplitude over the two incident field directions assuming that all frequencies are present with the same amplitude. The image obtained from the timedependent pulses is smoother and has fewer local maxima that could frustrate subsequent image processing algorithms for finding the minimum curve. The figures illustrate the value of a weighted average over straight averaging. When one synthesizes multi-frequency data without consideration of what kind of time signal the frequencies represent, one has no indication of how to weight the frequencies when averaging the data.

To give some idea of the computational intensity of these experiments, the forward solution for the time-dependent scattered field calculated on the $80 \times 80$ Cartesian grid via boundary integral techniques took 206 seconds using MATLAB with the DP Toolbox [18] on a parallel cluster of 6 Linux PC's (2 Ghz, 1280 MB RAM). In contrast, the inverse solution, calculated on a polar grid of 6400 evenly spaced points from 80 simulated far field measurements, took 13 seconds.

\section{References}

[1] Cakoni, F. and Colton, D. and Monk P., The direct and inverse scattering problems for partially coated obstacles, Inverse Problems, 17:1997-2015, 2001.

[2] D. Colton and A. Kirsch. A simple method for solving inverse scattering problems in the resonance region. Inverse Problems, 12(4):383-93, 1996.

[3] D. Colton and R. Kress. Inverse Acoustic and Electromagnetic Scattering Theory. Springer-Verlag, New York, 2nd edition, 1998. 


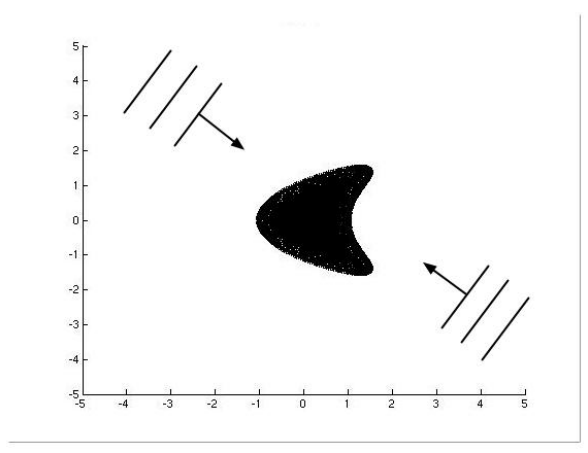

Figure 2: Sound-soft obstacles.

[4] D. Colton and P. Monk. A novel method for solving the inverse scattering problem for time-harmonic waves in the resonance region. SIAM J. Appl. Math., 45:1039-53, 1985.

[5] D. Colton and P. Monk. A novel method for solving the inverse scattering problem for time-harmonic waves in the resonance region II. SIAM J. Appl. Math., 46:506-23, 1986.

[6] G. Gilbarg and N. S. Trudinger. Elliptic Partial Differential Equations of Second Order. Springer-Verlag, Berlin, 1998.

[7] M. Ikehata. Reconstruction of an obstacle from the scattering amplitude at a fixed frequency. Inverse Problems, 14:949-54, 1998.

[8] A. Kirsch. Characterization of the shape of a scattering obstacle using the spectral data of the far field operator. Inverse Problems, 14:1489-1512, 1998.

[9] T. W. K. Koerner. Fourier Analysis. Cambridge University Press, Cambridge, 1988.

[10] R. Kress. Integral equation methods in inverse acoustic and electromagnetic scattering. In D. B. Ingham and L. C. Wrobel, editors, Boundary Integral Formulations for Inverse Analysis, pages 67-92. Computational Mechanics Publications, South Hampton, UK, 1997.

[11] P. D. Lax and R. S. Phillips. Scattering Theory. Academic Press, Toronto, Revised edition, 1989.

[12] R. Leis. Initial Boundary Value Problems in Mathematical Physics. B.G. Teubner, Stuttgart, 1986.

[13] D. R. Luke. Image synthesis for inverse obstacle scattering using the eigenfunction expansion theorem. Computing, Digital Object Identifier (DOI) 10.1007/s00607-0040096-9, 2004. 


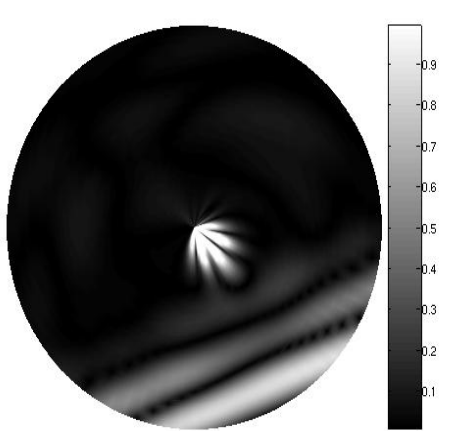

(a)

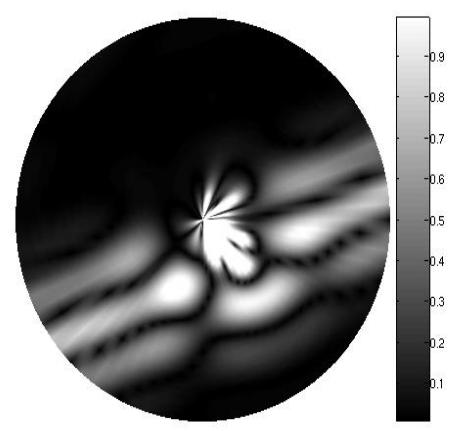

(b)

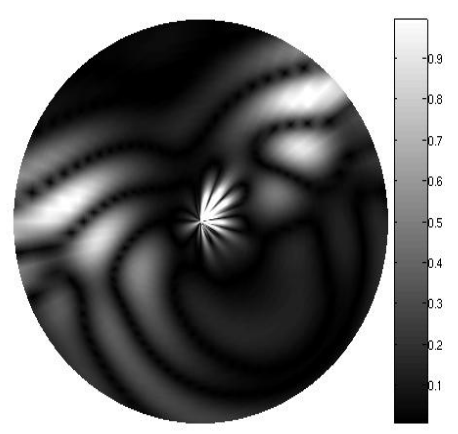

(c) (d)
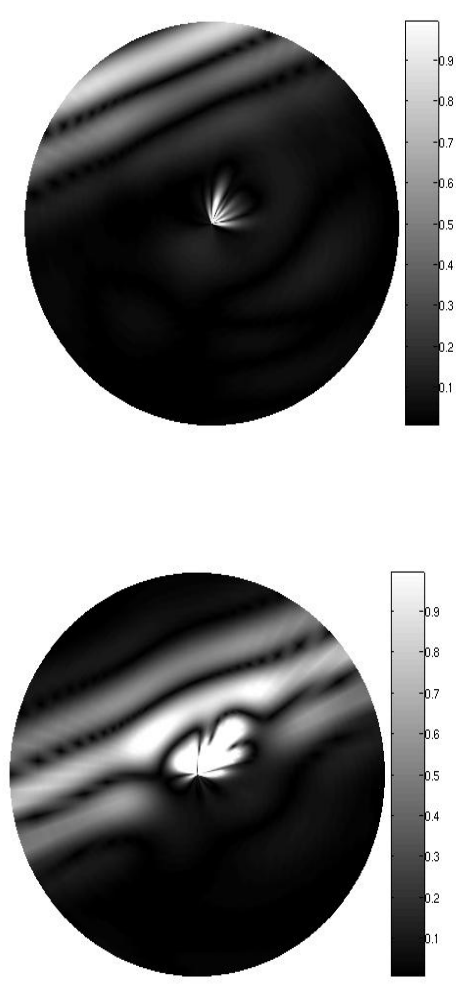

(e)

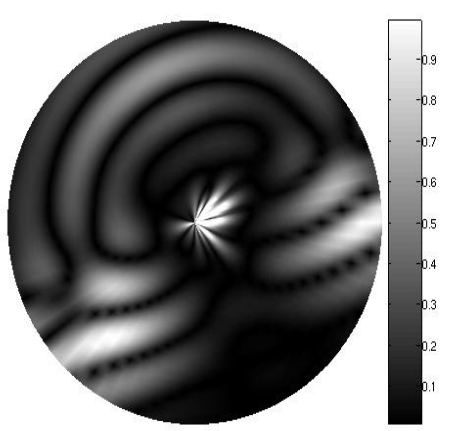

(f)

Figure 3: (a)-(c) Amplitude of the total wave calculated via the point-source method from far field measurements at times $t=21,31$ and 42 with an incident wave traveling southeast to northwest. (d)-(f) Amplitude of the total wave calculated via the point-source method from far field measurements at times $t=21,31$ and 42 with an incident wave traveling northwest to southeast. 
(a)

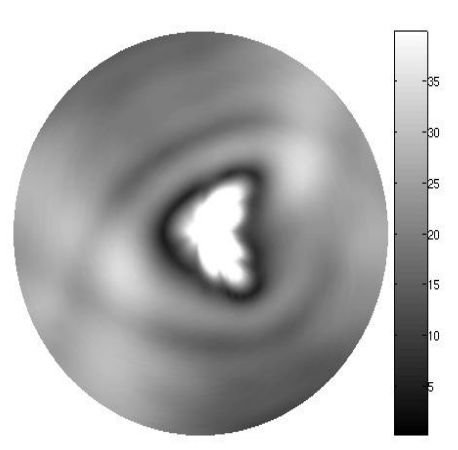

(e)

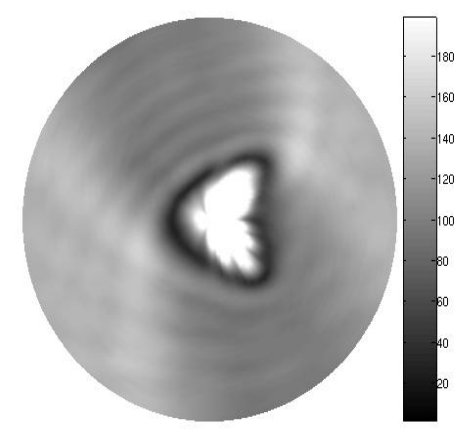

Figure 4: (a) Total field amplitude averaged over all time samples and incident pulse directions.

(b) Total field amplitude averaged over all frequencies for equal amplitude time-harmonic fields.

[14] D. R. Luke. Multifrequency inverse obstacle scattering: the point source method and generalized filtered backprojeciton. Math. and Computers in Simulation, 66(4-5):297$314,2004$.

[15] D. R. Luke and R. Potthast. The point source method in acoustic scattering: numerical reconstruction of the scattered field from far field measurements of inhomogeneous media. In IEEE 2002 International Conference on Acoustics, Speech and Signal Processing, pages IV-3541-IV-3544, Orlando, May 2002. IEEE.

[16] D. R. Luke and R. Potthast. The no response test - a sampling method for inverse scattering problems. SIAM J. Appl. Math., 63(4):1292-1312, 2003.

[17] C. S. Morawetz. The limiting amplitude principle. Comm. Pure Appl. Math., 15:349361, 1962.

[18] S. Pawletta, A. Westphal, W. Drewelow, P. Duenow, T. Pawletta, and R. Fink. Distributed and Parallel Application Toolbox (DP Toolbox). University of Rostock, http://www-at.e-technik.uni-rostock.de/rg_ac/dp/, 2003.

[19] R. Potthast. A fast new method to solve inverse scattering problems. Inverse Problems, 12:731-42, 1996.

[20] R. Potthast. A point-source method method for inverse acoustic and electromagnetic obstacle scattering problems. IMA Jour. Appl. Math., 61:119-40, 1998.

[21] R. Potthast. Point Sources and Multipoles in Inverse Scattering Theory. Chapman \& Hall, London, 2001.

[22] R. Potthast, J. Sylvester, and S. Kusiak. A 'range test' for determining scatterers with unknown physical properties. Inverse Problems, 19(3):533-547, 2003. 
[23] A. G. Ramm. Scattering by Obstacles. D. Reidel Publishing, Dordrecht, Holland, 1986.

[24] W. Rudin. Real and Complex Analysis. McGraw-Hill, 2nd edition, 1974. 